EESTI NSV TEADUSTE AKADEEMIA TOIMETISED. 28. KÖIDE

FOOSIKA * MATEMAATIKA. 1979, NR. 4

ИЗВЕСТИЯ АКАДЕМИИ НАУК ЭСТОНСКОП ССР. ТОМ 28

ФИЗИКА * МАТЕМАТИКА. 1979, № 4

\title{
ИНФОРМАЦИОННАЯ ХАРАКТЕРИСТИКА СИСТЕМЫ ПАР РАЗБИЕНИЙ НА КОНЕЧНОМ МНОЖЕСТВЕ
}

\author{
(Представлена И. Эпиком)
}

В последнее время в связи с декомпозицией сложных систем возрос интерес к применению информационных критериев для оценки различных вариантов декомпозиций $\left[{ }^{1,2}\right]$. Несмотря на различие в практических целях $\left[{ }^{1-3}\right]$, в общем все эти критерии основываются на т. н. свойстве субаддитивности энтропии для разбиений системы, которое состоит в том, что энтропия системы никогда не превышает суммарной энтропии ее подсистем. Благодаря свойству субаддитивности разницу между суммарной энтропией подсистем и энтропией системы можно использовать для оценки информационной зависимости выбранного класса подсистем, ибо она всегда положительна и при полной независимости подсистем равняется нулю.

В настоящей работе рассматривается обмен информации в системе пар разбиений на конечном базисном множестве. Эту систему можно интерпретировать как некоторый дискретный преобразователь информации со многими входами и выходами, где каждая пара разбиений представляет собой информационный канал от входа к выходу преобразователя, а базисное множество есть множество выходных символов некоторого стационарного источника сообщений. Такой подход удобен при оценке сложности различных вариантов декомпозиции дискретных преобразователей информации. Полезность использования пар разбиений при декомпозиции автоматов впервые показали Дж. Хартманис и Р. Е. Стирнз $\left[{ }^{4}\right]$. К сожалению, они ограничили свой подход только качественной стороной вопроса, не применив нигде количественных соотношений. Настоящий подход можно рассматривать как продолжение их исследования в том смысле, что здесь находятся количественные информационные характеристики для пар разбиений. Это позволяет оценивать и системы несравнимых пар разбиений.

Основные результаты работы:

1. Свойство субаддитивности справедливо и для системы пар разбиений.

2. Суммарное количество информации, которой обмениваются различные пары разбиений, равняется разнице между суммарной энтропией всех пар разбиений и энтропией их произведения.

Пусть дано конечное множество $X=\left\{x_{1}, x_{2}, \ldots, x_{n}\right\}$ при заданном распределении вероятностей $P(X)$ на нем, т. е. задана функция $p: X \rightarrow R^{+}$из множества $X$ во множество положительных действитель- 
ных чисел, такое, что $\sum_{i=1}^{n} p\left(x_{i}\right)=1$. Разбиение множества $X$ на непересекающиеся подмножества (блоки) $B_{i}^{(1)}, B_{i}^{(2)}, \ldots, B_{i}^{(\alpha)}, \ldots, B_{i}^{(n t)}$ обозначим через $\pi_{i}(X)$. В частности, нулевое разбиение (т. е. разбиение, каждый блок которого содержит лишь один элемент из $X$ ) будем обозначать через $0_{x}$, а единичное (т. е. разбиение, содержащее только один блок) - через $1_{x}$. Для любых разбиений $\pi_{i}(X)$ и $\pi_{k}(X)$ примем, что

$$
\pi_{i} \cdot \pi_{k} \underset{\mathrm{Df}}{=}\left\{B_{i}^{(\alpha)} \cap B_{k}^{(\beta)} \mid B_{i}^{(\alpha)} \in \pi_{i} \wedge B_{k}^{(\beta)} \in \pi_{k}\right\}
$$

и

$$
\pi_{i} \leqslant \pi_{k} \underset{\mathrm{Df}}{\Longleftrightarrow} \pi_{i} \cdot \pi_{k}=\pi_{i}
$$
$\left[{ }^{5,6}\right]$ :

Определим теперь для каждого разбиения $\pi_{i}(X)$ энтропию $H\left(\pi_{i}\right)$

$$
H\left(\pi_{i}\right) \underset{\mathrm{Df}}{=}-\sum_{\alpha=1}^{n_{t}} p\left(B_{i}^{(\alpha)}\right) \ln p\left(B_{i}^{(\alpha)}\right)
$$

где

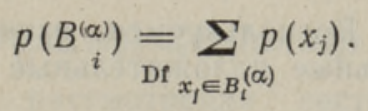

Энтропию множества $X$ определим как энтропию нулевого разбиения, T. e.

$$
H(X) \underset{\mathrm{Df}}{=} H\left(0_{x}\right)=-\sum_{j=1}^{n} p\left(x_{j}\right) \ln p\left(x_{j}\right) .
$$

Любую пару разбиений $\left\langle\pi_{i}(X), \pi_{k}(X)\right\rangle$ будем называть каналом. Для каждого канала $\left\langle\pi_{i}, \pi_{k}\right\rangle$ определим энтропию $H\left(\left\langle\pi_{i}, \pi_{k}\right\rangle\right)$ :

$$
H\left(\left\langle\pi_{i}, \pi_{k}\right\rangle\right) \underset{\mathrm{Df}}{=} H\left(\pi_{k} / \pi_{i}\right)=-\sum_{\alpha=1}^{n_{t}} p\left(B_{i}^{(\alpha)}\right) \sum_{\beta=1}^{n_{k}} p\left(B_{k}^{(\beta)} / B_{i}^{(\alpha)}\right) \ln p\left(B_{k}^{(\beta)} / B_{i}^{(\alpha)}\right),
$$

где

$$
p\left(B_{k}^{(\beta)} / B_{i}^{(\alpha)}\right) \underset{\text { Df }}{=} p\left(B_{i}^{(\alpha)}, B_{k}^{(\beta)}\right) / p\left(B_{i}^{(\alpha)}\right)
$$

Для любых каналов $\left\langle\pi_{h}, \pi_{i}\right\rangle$ и $\left\langle\pi_{j}, \pi_{k}\right\rangle$ положим

$$
\left\langle\pi_{h}, \pi_{i}\right\rangle \geqslant\left\langle\pi_{j}, \pi_{k}\right\rangle \underset{\mathrm{Df}}{\Longleftrightarrow}\left(\pi_{h} \geqslant \pi_{j} \bigwedge \pi_{i} \geqslant \pi_{k}\right)
$$

и

$$
\left\langle\pi_{h}, \pi_{i}\right\rangle \cdot\left\langle\pi_{j}, \pi_{k}\right\rangle=\underset{\mathrm{Df}}{=}\left\langle\pi_{h} \cdot \pi_{j}, \pi_{i} \cdot \pi_{k}\right\rangle .
$$

Пусть $\pi_{i}(X)$ и $\pi_{k}(X)$ - произвольные разбиения. Тогда непосредственно из определения понятия энтропии для канала следует, что:

a) $H\left(\left\langle\pi_{i}, \pi_{k}\right\rangle\right) \geqslant 0$,

б) $H\left(\left\langle 0_{x}, \pi_{i}\right\rangle\right)=H\left(\left\langle\pi_{i}, \pi_{i}\right\rangle\right)=H\left(\left\langle\pi_{i}, 1_{x}\right\rangle\right)=0$,

в) $H\left(\left\langle 1_{x}, \pi_{i}\right\rangle\right)=H(X)-H\left(\left\langle\pi_{i}, 0_{x}\right\rangle\right)=H\left(\pi_{i}\right)$. 
Л е м м а 1. Для любого канала $\left\langle\pi_{i}, \pi_{k}\right\rangle$ справедливо

$$
H\left(\left\langle\pi_{i}, \pi_{k}\right\rangle\right)=H\left(\pi_{i} \cdot \pi_{k}\right)-H\left(\pi_{i}\right) .
$$

Д о к а з т ель с в о. Действительно, по определению

$$
\begin{gathered}
H\left(\left\langle\pi_{i}, \pi_{k}\right\rangle\right)=-\sum_{\alpha, \beta=1}^{n_{l}, n_{k}} p\left(B_{i}^{(\alpha)}, B_{k}^{(\beta)}\right) \ln \left(p\left(B_{i}^{(\alpha)}, B_{k}^{(\beta)}\right) / p\left(B_{i}^{(\alpha)}\right)\right)= \\
=-\sum_{\alpha, \beta=1}^{n_{l}, n_{k}} p\left(B_{i}^{(\alpha)}, B_{k}^{(\beta)}\right) \ln \left(B_{i}^{(\alpha)}, B_{k}^{(\beta)}\right)+ \\
+\sum_{\alpha=1}^{n_{t}} p\left(B_{i}^{(\alpha)}\right) \ln p\left(B_{i}^{(\alpha)}\right)\left(\sum_{\beta=1}^{n_{k}} p\left(B_{k}^{(\beta)} / B_{i}^{(\alpha)}\right)\right)=H\left(\pi_{i} \cdot \pi_{k}\right)-H\left(\pi_{i}\right) .
\end{gathered}
$$

Из леммы 1 следует, что если $\pi_{k} \geqslant \pi_{i}$, то $H\left(\left\langle\pi_{i}, \pi_{k}\right\rangle\right)=0$.

Л е м а 2. Для любых каналов $\left\langle\pi_{h}, \pi_{i}\right\rangle u\left\langle\pi_{j}, \pi_{k}\right\rangle$ при произвольном распределении вероятностей $P(X)$ имеет место

$$
H\left(\left\langle\pi_{h}, \pi_{i}\right\rangle\right)+H\left(\left\langle\pi_{j}, \pi_{k}\right\rangle\right) \geqslant H\left(\left\langle\pi_{h}, \pi_{i}\right\rangle \cdot\left\langle\pi_{j}, \pi_{k}\right\rangle\right) .
$$

Д ок а з а тельст во. Воспользуемся известным фактом $\left[{ }^{7}\right]$, что если $p_{j}$ и $q_{j}$-произвольные положительные числа, удовлетворяющие равенствам

To

$$
\sum_{j=1}^{m} p_{j}=\sum_{j=1}^{m} q_{j}=1
$$

$$
\sum_{j=1}^{m} q_{j} \ln q_{j} \geqslant \sum_{j=1}^{m} q_{j} \ln p_{j}
$$

Умножая выражения для энтропий $H\left(\left\langle\pi_{h}, \pi_{i}\right\rangle\right)$ и $H\left(\left\langle\pi_{j}, \pi_{k}\right\rangle\right)$ соответственно на единицы вида

$$
1=\sum_{\gamma, \delta=1}^{n_{f}, n_{k}} p\left(\left(B_{j}^{(\gamma)}, B_{k}^{(\delta)}\right) /\left(B_{h}^{(\alpha)}, B_{i}^{(\beta)}\right)\right)=\sum_{\alpha, \beta=1}^{n_{n}, n_{t}} p\left(\left(B_{h}^{(\alpha)}, B_{i}^{(\beta)}\right) /\left(B_{j}^{(\gamma)}, B_{k}^{(\delta)}\right)\right),
$$

получаем

и

$$
H\left(\left\langle\pi_{h}, \pi_{i}\right\rangle\right)=-\sum_{\alpha, \beta, \gamma, \delta=1}^{n_{h}, n_{l}, n_{l}, n_{k}} p\left(B_{h}^{(\alpha)}, B_{i}^{(\beta)}, B_{j}^{(\gamma)}, B_{k}^{(\delta)}\right) \ln \left(p\left(B_{h}^{(\alpha)}, B_{i}^{(\beta)}\right) / p\left(B_{h}^{(\alpha)}\right)\right)
$$

$$
H\left(\left\langle\pi_{j}, \pi_{k}\right\rangle\right)=-\sum_{\alpha, \beta, \gamma, \delta=1}^{n_{h}, n_{t}, n_{t}, n_{k}} p\left(B_{h}^{(\alpha)}, B_{i}^{(\beta)}, B_{j}^{(\gamma)}, B_{k}^{(\delta)}\right) \ln \left(p\left(B_{j}^{(\gamma)}, B_{k}^{(\delta)}\right) / p\left(B_{j}^{(\gamma)}\right)\right) .
$$

Положим теперь

$$
\begin{gathered}
n=n_{h} n_{i}, \quad m=n_{j} n_{k}, \quad r_{u}=p\left(B_{h}^{(\alpha)}, B_{j}^{(\gamma)}\right), \\
p_{u v}=p\left(\left(B_{i}^{(\beta)}, B_{k}^{(\delta)}\right) /\left(B_{h}^{(\alpha)}, B_{j}^{(\gamma)}\right)\right), \\
q_{u v}=p\left(B_{i}^{(\beta)} / B_{h}^{(\alpha)}\right) p\left(B_{k}^{(\delta)} / B_{j}^{(\gamma)}\right) .
\end{gathered}
$$

Тогда 


$$
H\left(\left\langle\pi_{h}, \pi_{i}\right\rangle\right)+H\left(\left\langle\pi_{j}, \pi_{k}\right\rangle\right)=-\sum_{u=1}^{n} r_{u} \sum_{v=1}^{m} p_{u v} \ln q_{u v}
$$

и

$$
H\left(\left\langle\pi_{h}, \pi_{i}\right\rangle \cdot\left\langle\pi_{j}, \pi_{k}\right\rangle\right)=-\sum_{u=1}^{n} r_{u} \sum_{v=1}^{m} p_{u v} \ln p_{u v}
$$

Но так как $\sum_{v=1}^{m} p_{u v}=\sum_{v=1}^{m} q_{u v}=1$, то, используя неравенство (1), заключаем, что

$$
H\left(\left\langle\pi_{h}, \pi_{i}\right\rangle\right)+H\left(\left\langle\pi_{j}, \pi_{k}\right\rangle\right) \geqslant H\left(\left\langle\pi_{h}, \pi_{i}\right\rangle \cdot\left\langle\pi_{j}, \pi_{k}\right\rangle\right) .
$$

Из леммы 2 вытекает, что для любых разбиений $\pi_{i}(X)$ и $\pi_{k}(X)$ справедливо

и

$$
H\left(\pi_{i}\right)+H\left(\pi_{k}\right) \geqslant H\left(\pi_{i} \cdot \pi_{k}\right)
$$

$$
H\left(\pi_{k}\right) \geqslant H\left(\left\langle\pi_{i}, \pi_{k}\right\rangle\right) .
$$

Можно показать также, что если $\pi_{j}(X) \geqslant \pi_{h}(X)$ и $\pi_{i}(X) \geqslant \pi_{k}(X)$, то при произвольном распределении вероятностей $P(X)$

$$
H\left(\left\{\pi_{h}, \pi_{i}\right\rangle\right) \leqslant H\left(\left\langle\pi_{j}, \pi_{k}\right\rangle\right) .
$$

В дальнейшем при рассмотрении системы каналов $K(X)=$ $=\left\{\left\langle\pi^{\prime}{ }_{1}, \pi^{\prime \prime}{ }_{1}\right\rangle,\left\langle\pi^{\prime}{ }_{2}, \pi^{\prime \prime}{ }_{2}\right\rangle, \ldots,\left\langle\pi^{\prime}{ }_{i}, \pi^{\prime \prime}{ }_{i}\right\rangle, \ldots,\left\langle\pi^{\prime}{ }_{w}, \pi^{\prime \prime}{ }_{w}\right\rangle\right\}$ на $X$ будем пользоваться обозначением $\mathrm{Q}_{i}=\underset{\mathrm{Df}}{=}\left\langle\boldsymbol{\pi}^{\prime}{ }_{i}, \pi^{\prime \prime}{ }_{i}\right\rangle$. Для любой системы каналов $K(X)$ примем, что $m(K) \underset{\text { Df }}{=} \prod_{p_{t} \in K} \mathrm{Q}_{i}$ и $H(K) \underset{\text { Df }}{=} H(m(K))$. Используя математическую индукцию, получим из леммы 2 следующий результат.

Т еорем а 1. Для любой системь каналов $K$ справедливо неравенство

$$
\sum_{\rho_{t} \in K} H\left(\varrho_{i}\right) \geqslant H(K) .
$$

Условная энтропия для любых каналов $\varrho_{i}$ и $\varrho_{h}$ имеет вид

$$
H\left(\varrho_{i} / \varrho_{h}\right) \underset{\text { Df }}{=} H\left(\varrho_{h} \cdot \varrho_{i}\right)-H\left(\varrho_{h}\right) .
$$

Пусть $\varrho_{i}, \varrho_{j}$ и $\varrho_{k}$ - произвольные каналы на $X$. Определим теперь количество информации, которой обмениваются каналы $\varrho_{j}$ и $\varrho_{k}$ при известном канале 2 в в виде следующей разности:

$$
I\left(\varrho_{j} \rightarrow \varrho_{k} \mid \varrho_{i}\right) \underset{\text { Df }}{=} H\left(\varrho_{k} / \varrho_{i}\right)-H\left(\varrho_{k} / \varrho_{i} \cdot \varrho_{j}\right) .
$$

Нетрудно показать, что для любых каналов $Q_{i}, Q_{j}$ и $Q_{k}$ $I\left(\varrho_{j} \rightarrow \varrho_{k} \mid \varrho_{i}\right)=I\left(\varrho_{k} \rightarrow \varrho_{j} \mid \varrho_{i}\right)=I\left(\pi_{j}^{\prime} \cdot \pi_{j}^{\prime \prime} \rightarrow \pi_{k}^{\prime} \cdot \pi_{k}^{\prime \prime} \mid \pi_{i}^{\prime} \cdot \pi_{i}^{\prime \prime}\right)-I\left(\pi_{j}^{\prime} \rightarrow \pi_{k}^{\prime} \mid \pi_{i}^{\prime}\right)$.

Пусть $\varrho_{\omega}(X)$ - произвольный канал, а $K(X)=\left\{\varrho_{1}, \varrho_{2}, \ldots, \varrho_{w}\right\}-$ произвольная система каналов на $X$. Легко видеть, что

$$
\sum_{i=1}^{w} I\left(\varrho_{i} \rightarrow \varrho_{\omega} \mid \varrho_{1} \cdot \varrho_{2} \cdot \ldots \cdot \varrho_{i-1}\right)=H\left(\varrho_{\omega}\right)-H\left(\varrho_{\omega} / m(K)\right) .
$$

Поэтому, если $\mathrm{Q}_{\omega} \geqslant m(K)$, то 


$$
\sum_{i=1}^{w} I\left(\varrho_{i} \rightarrow \varrho_{\omega} \mid \varrho_{1} \cdot \varrho_{2} \cdot \ldots \cdot \varrho_{i-1}\right)=H\left(\varrho_{\omega}\right) .
$$

Это значит, что суммарное количество информации, поочередно передаваемой каналами из произвольной системы каналов $K(X)$, не зависит от упорядочений этих каналов и при $\varrho_{\omega} \leqslant m(K)$ всегда равняется $H\left(\varrho_{\omega}\right)$. В дальнейшем при $I\left(\varrho h \rightarrow \varrho \omega \mid \varrho i_{1} \cdot \varrho i_{2} \cdot \ldots \cdot \varrho i_{k}\right)$, если не оговорено иначе, будем всегда предполагать, что $i_{1}<i_{2}<\ldots<i_{k}$ и $h \neq$ $\neq i_{1}, i_{2}, \ldots, i_{k}$. Ясно, что в общем $I\left(\varrho_{h} \rightarrow \varrho_{\omega} \mid \varrho_{i_{1}} \cdot \varrho_{i_{2}} \cdot \ldots \cdot \mathrm{Q}_{i_{s}}\right) \neq$ $\neq I\left(\varrho_{h} \rightarrow \varrho_{\omega} \mid \varrho_{j_{1}} \cdot \varrho_{j_{2}} \cdot \ldots \varrho_{j_{t}}\right)$, т. е. количество информации, которой обмениваются каналы $\varrho_{h} \in K(X)$ и $\varrho_{\varrho}$, зависит от упорядочений системы $K$. Рассмотрим далее среднее количество информации $I\left(\varrho_{h} \rightarrow \varrho_{\omega}\right)$, которой обмениваются каналы $\mathrm{Q}_{h} \in K(X)$ и $\mathrm{Q}_{\omega}$, относительно всех возможных упорядочений системы $K$.

Нетрудно видеть, что

$$
\begin{aligned}
\bar{I}\left(\varrho_{h} \rightarrow \varrho_{\omega}\right)= & \sum_{k=0}^{w-1} \sum_{i_{1}, i_{2}, \ldots, i_{k}=1}^{w} p\left(I\left(\varrho_{h} \rightarrow \varrho_{\omega} \mid \varrho_{i_{1}} \cdot \varrho_{i_{2}} \cdot \ldots \cdot \varrho_{i_{k}}\right)\right) \times \\
& X I\left(\varrho_{h} \rightarrow \varrho_{\omega} \mid \varrho_{i_{1}} \cdot \varrho_{i_{2}} \cdot \ldots \cdot \varrho_{i_{k}}\right)
\end{aligned}
$$

где

$$
p\left(I ( \varrho _ { h } \rightarrow \varrho _ { \omega } | \varrho _ { i _ { 1 } } \cdot \varrho _ { i _ { 2 } } \cdot \ldots \cdot \varrho _ { i _ { k } } ) \underset { \text { Df } } { = } \left\{\begin{array}{cc}
k !(w-k-1) ! / w !, & \text { если } k \geqslant 1 ; \\
1 / w, & \text { если } k=0 .
\end{array}\right.\right.
$$

Можно показать, что для любых $\mathrm{Q}_{i}, \mathrm{Q}_{k} \in K$

$$
\bar{I}\left(\varrho_{i} \rightarrow \varrho_{k}\right)=\bar{I}\left(\varrho_{k} \rightarrow \varrho_{i}\right) .
$$

Учитывая, что суммарное количество информации, которой поочередно обмениваются, с одной стороны, каналы из произвольной системы каналов $K$ и, с другой стороны, какой-то канал @ю, не зависит от упорядочений этих каналов, легко убедиться в справедливости следующей леммы.

Л ем м а 3. Для любой системы каналов $K(X)$ и канала @ при произвольном распределении вероятностей $P(X)$ верно

$$
\sum_{\rho_{h} \in K} \bar{I}\left(\varrho_{h} \rightarrow \varrho_{\omega}\right)=H\left(\varrho_{\omega}\right)-H\left(\varrho_{\omega} / m(K)\right) .
$$

Из леммы 3 следует, что для любой системы каналов $K(X)$ справедливо

$$
\sum_{\rho_{t}, \rho_{j} \in K} \bar{I}\left(\varrho_{i} \rightarrow \varrho_{j}\right)=\sum_{\rho_{h} \in K} H\left(\varrho_{h}\right) .
$$

Введем обозначение

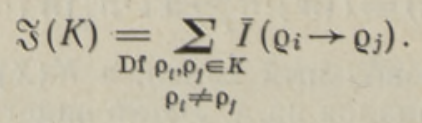

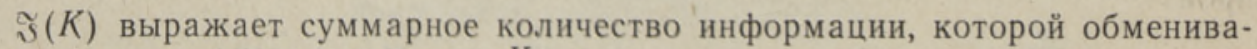
ются различные каналы из $K$.

Т е о рем а 2. Для любой системь каналов $K=\left\{\varrho_{1}, \varrho_{2}, \ldots, \varrho_{w}\right\}$ справедливо 


$$
\mathfrak{\Im}(K)=\sum_{j=1}^{w} H\left(\varrho_{j}\right)-H(K) .
$$

Дока за тельство. Так как $\sum_{h, i=1}^{w} \bar{I}\left(\varrho_{h} \rightarrow \varrho_{i}\right)=\sum_{j=1}^{w} H\left(\varrho_{j}\right)$, то

$$
\mathfrak{\Im}(K)=\sum_{h, i=1}^{w} \bar{I}\left(\varrho_{h} \rightarrow \varrho_{i}\right)-\sum_{j=1}^{w} \bar{I}\left(\varrho_{j} \rightarrow \varrho_{j}\right)=\sum_{k=1}^{w} H\left(\varrho_{k}\right)-\sum_{j=1}^{w} \bar{I}\left(\varrho_{j} \rightarrow \varrho_{j}\right) .
$$

Но по определению

$$
\begin{gathered}
\bar{I}\left(\varrho_{j} \rightarrow \varrho_{j}\right)=\sum_{k=0}^{w-1} \sum_{i_{1}, i_{2}, \ldots, i_{k}=1}^{w} p\left(I\left(\varrho_{j} \rightarrow \varrho_{j} \mid \varrho_{i_{1}} \cdot \varrho_{i_{2}} \cdot \ldots \cdot \varrho_{i_{k}}\right) \times\right. \\
\quad \times\left[H\left(\varrho_{j} \cdot \varrho_{i_{1}} \cdot \varrho_{i_{2}} \cdot \ldots \cdot \varrho_{i_{k}}\right)-H\left(\varrho_{i_{1}} \cdot \varrho_{i_{2}} \cdot \ldots \cdot \varrho_{i_{k}}\right)\right] .
\end{gathered}
$$

Поэтому

$$
\begin{aligned}
\sum_{j=1}^{w} \bar{I}\left(\varrho_{j} \rightarrow \varrho_{j}\right)=\sum_{j=1}^{w} \sum_{k=0}^{w-1} \sum_{i_{1}, i_{2}, \ldots,}^{w} \sum_{i_{k}=1}^{w} p\left(I\left(\varrho_{j} \rightarrow \varrho_{j} \mid \varrho_{i_{1}} \cdot \varrho_{i_{2}} \cdot \ldots \cdot \varrho_{i_{k}}\right) \times\right. \\
\times\left[H\left(\varrho_{j} \cdot \varrho_{i_{1}} \cdot \varrho_{i_{2}} \cdot \ldots \cdot \varrho_{i_{k}}\right)-H\left(\varrho_{i_{1}} \cdot \varrho_{i_{2}} \cdot \ldots \cdot \varrho_{i_{k}}\right)\right] .
\end{aligned}
$$

Разложив последнее выражение по переменной $k$, с учетом

$$
\sum_{\substack{i_{1}, i_{2}, \ldots, \ldots, i_{k}=1 \\ i_{1} \neq i_{2}, i_{3}, \ldots, i_{k} \\ i_{2}<i_{3}<\ldots<i_{k}}}^{w} H\left(\varrho_{i_{1}} \cdot \varrho_{i_{2}} \cdot \ldots \cdot Q_{i k}\right)=k \sum_{\substack{i_{1}, i_{2}, \ldots, i_{k}=1 \\ i_{1}<i_{2}<\ldots<i_{k}}}^{w} H\left(\varrho_{i_{1}} \cdot \varrho_{i_{2}} \cdot \ldots \cdot \varrho_{i_{k}}\right)
$$

получим

$$
\bar{I}\left(\varrho_{j} \rightarrow \varrho_{j}\right)=H\left(\prod_{i=1}^{w} \varrho_{i}\right)=H(K) .
$$

Итак,

$$
\mathfrak{J}(K)=\sum_{k=1}^{w} H\left(\varrho_{k}\right)-\sum_{j=1}^{w} \bar{I}\left(\varrho_{j} \rightarrow \varrho_{j}\right)=\sum_{k=1}^{w} H\left(\varrho_{k}\right)-H(K),
$$

что и требовалось доказать.

Из теоремы 2 получаем для $\Im(K)$ следующую оценку:

$$
0 \leqslant \widetilde{J}(K) \leqslant \sum_{\rho_{t} \in K} H\left(\varrho_{i}\right) .
$$

Для информационной характеристики системы каналов $K$ воспользуемся понятием информационной связки $\mathfrak{B}(K)$, которая определяется так:

$$
\mathfrak{B}(K) \underset{\mathrm{Df}}{=} \max _{P(\boldsymbol{X})} \mathfrak{\Im}(K),
$$

где максимум берется по всем возможным распределениям вероятности $P(X)$.

Приме р. Воспользуемся понятием информационной связки для оценки сложности булевых функций, предполагая, что функция тем сложнее, чем больше ее информационная связка. При рассмотрении сложности $m$-переменной булевой функции $F$ применим следующую интерпретацию для приведенных выше формальных понятий: 
1) множество $X=\left\{x_{1}, x_{2}, \ldots, x_{2}^{m}\right\}$ есть множество всех возможных комбинаций входных переменных $y_{1}, y_{2}, \ldots, y_{m}$ функции $F$;

2) $K_{F}=\left\{\left\langle\pi_{i}, \pi_{F}\right\rangle \mid i=1,2, \ldots, m\right\}$, где $\pi_{i}$ - разбиение, индуцированное переменной $y_{i}$, а $\pi_{F}-$ разбиение, индуцированное значениями функции $F$.

\begin{tabular}{|c|c|c|c|}
\cline { 2 - 4 } & $y_{1}$ & $y_{2}$ & $F\left(y_{1}, y_{2}\right)$ \\
\hline$x_{1}$ & 0 & 0 & 1 \\
\hline$x_{2}$ & 0 & 1 & 0 \\
\hline$x_{3}$ & 1 & 0 & 0 \\
\hline$x_{4}$ & 1 & 1 & 0 \\
\hline
\end{tabular}

Например, если функция $F\left(y_{1}, y_{2}\right)$ дана в виде приведенной таблицы, то $X=\left\{x_{1}, x_{2}, x_{3}, x_{4}\right\}$,

$$
\begin{array}{ll}
\pi_{1}=\left\{\overline{x_{1} x_{2}}, \overline{x_{3} x_{4}}\right\}, \quad \pi_{2}=\left\{\overline{x_{1} x_{3}}, \overline{x_{2} x_{4}}\right\}, \\
\pi_{F}=\left\{\bar{x}_{1}, \overline{x_{2} x_{3} x_{4}}\right\}, \quad K_{F}=\left\{\left\langle\pi_{1}, \pi_{F}\right\rangle,\left\langle\pi_{2}, \pi_{F}\right\rangle\right\} .
\end{array}
$$

Для функции $F$ получаем, что

$$
\begin{gathered}
\mathfrak{B}\left(K_{F}\right)=\max _{P(X)}\left(H\left(\left\langle\pi_{1}, \pi_{F}\right\rangle\right)+H\left(\left\langle\pi_{2}, \pi_{F}\right\rangle\right)-\right. \\
\left.-H\left(\left\langle\pi_{1}, \pi_{F}\right\rangle \cdot\left\langle\pi_{2}, \pi_{F}\right\rangle\right)\right)=0,962 \text { нат. }
\end{gathered}
$$

\section{ЛИТ Е РА Т У Р А}

1. Кен ге р ли н ски й Г. А., Изв. АН СССР, Техн, кибернетика, № 1, 121-128 (1978).

2. Боголюбов И. Н., В ор онцов а И. П., О вси евич Б. Л., Изв. АН СССР, Техн. кибернетика, № 3, 150-155 (1974).

3. W a t a n a b e, S., IBM J. Res. Develop., 4, № 1, 66-82 (1960).

4. Hartmanis, J., Stearns, R. E., Algebraic structure theory of sequential machines, Prentice-Hall, Inc. Englewood Cliffs, New York, 1966.

5. Ш еннон К., Работы по теории информации и кибернетике, М., Изд-во иностр. лит., 1963.

6. Ф а н о Р., Передача информации, М., «Мир», 1965.

7. Бов бель Е. И., Д ан ей ко И. К., Изох В. В., Элементы теории информации, Минск, Изд. БГУ, 1974.

\section{Институт термофизики и электрофизики Академии наук Эстонской ССР}

\section{Поступила в редакцию} 18/VI 1979

\section{T. LAUSMAA}

\section{INFOSIDE LOPLIKUS TUKELDUSPAARIDE SUSTEEMIS}

On vaadeldud informatsioonivahetust lõplikul baashulgal moodustatud tükelduspaaride vahel. Selgub, et summaarne informatsioonihulk, mis vahetatakse erinevate tükelduspaaride vahel, vōrdub tükelduspaaride summaarse entroopia ning nende korrutise entroopia vahega. Saadud tulemus vōimaldab hinnata diskreetsete infomuundurite keerukust. 


\section{EXCHANGE OF INFORMATION IN THE SYSTEM OF PARTITION PAIRS ON A FINITE SET}

Lately, in connection with the decomposition of complicated systems the interest in informational criteria for estimating different possible ways of decomposition has grown $\left[{ }^{1,2}\right]$. Regardless of various practical objects $\left[{ }^{1-3}\right]$, all these criteria are mostly based on the property of «subadditivity», meaning that the entropy of any system is always not larger than the total entropy of its subsystems. In virtue of this property the difference between the total entropy of subsystems and the entropy of the given system is always non-negative and in the case of total independence of subsystems becomes zero and thus can be used for estimating the informational dependence of the selected set of subsystems.

In the present paper the exchange of information in the system of partition pairs on a finite basic set is considered. This set of partition pairs can be interpreted as a discrete infoconverter with a lot of inputs and outputs, assigning to each partition pair an infochannel from an input to an output. In this line one can interpret the basic finite set as a set of outputs of a stationary information source. This approach is convenient for estimating the complexity of different possible ways of decomposition of automata was first discussed by J. Hartmanis and R. E. Stearns $\left[{ }^{4}\right]$. Unfortunately their approach was only qualitive and no quantitive relations were given. The present paper provides quantitative informational measures for partition pairs thus enabling to estimate even the systems consisting of incomparable partition pairs.

The main results of this paper are:

1. The property of «subadditivity» holds in the system of partition pairs;

2. The total amount of information exchanged between different partition pairs is equal to the difference between the total amount of entropy of all these partition pairs and the entropy of its product. 